\title{
Noninvasive Respiratory Support in Pediatrics
}

\author{
Cesare Gregoretti, Maria Adele Figini, Fabrizio Racca \\ and Edoardo Calderini
}

\subsection{Introduction}

The conventional management of acute respiratory failure (ARF) consists of endotracheal intubation; this carries potential risks, including ventilator-associated pneumonia and laryngeal-tracheal damage [1,2]. Noninvasive respiratory support (NRS) is an alternative form of respiratory treatment which incorporates various techniques aimed at improving alveolar ventilation, oxygenation, and unloading of the respiratory muscles without the need for an invasive tracheal device. Because of its safety and effectiveness, the use of NRS has been adopted throughout the world. During the last 25 years, NRS techniques have increasingly been used in the treatment of both chronic respiratory failure and ARF in adult patients in several pathological conditions. NRS applied to adults in the acute setting has been found to improve outcome, reduce the rate of intubation, and decrease the rate of complications [3].

NRS includes noninvasive continuous positive airway pressure (NCPAP) and noninvasive positive pressure ventilation (NPPV) delivered through an interface (nasal/facial mask or helmet) and high-pressure freeflow gas and turbine or piston-driven ventilators [4]. Despite the lack of a full clinical picture, in recent years NRS has been increasingly used in pediatric intensive care units and emergency departments mainly because several uncontrolled clinical trials showed improved outcomes in selected patients with ARF when compared to standard treatment. At present, NRS in children with ARF is mainly performed by experienced centers, and no universally accepted guidelines have been proposed even outside the critical care area in less severe forms of respiratory insufficiency [2]. In a review published in 2001, the authors concluded that NRS may have limited benefits in a group of carefully selected pediatric patients with acute hypoxemic and hypercarbic forms of respiratory failure [5]. However, during the last few years, its use has increased and data supporting the use of this new technique in children are growing [6-10].

C. Gregoretti $(\square)$

Department of Anesthesiology and Intensive Care

Azienda Ospedaliera CTO-CRF-Maria Adelaide, Turin, Italy

e-mail: c.gregoretti@gmail.com 


\subsubsection{Rationale for Noninvasive Respiratory Support}

NRS, as mentioned previously, is the delivery of ventilatory support without the need for an invasive airway intervention procedure, such as endotracheal intubation or tracheotomy. The application of NRS to a patient can be "curative", as an alternative to endotracheal intubation once ARF occurs, or even "prophylactic", to prevent respiratory distress in patients who are at a higher risk of developing ARF (e.g., in postoperative and postextubation settings) or whenever the development of muscle weakness or fatigue is impending. Two types of NRS are most commonly used, i.e., NCPAP and NPPV.

The application of NCPAP takes place mainly through a high-pressure gas flow circuit, which comprises a gas circuit, a blender, a flow meter, and a positive endexpiratory pressure (PEEP) valve. Alternatively, a demand valve ventilator can be used and PEEP can be generated by high gas flows directed through a tube with increased resistance (the Coanda effect). NCPAP delivers a constant distending airway pressure throughout the entire respiratory cycle, while the patient is spontaneously breathing. It exerts its effects by: (1) increasing oxygenation and $\mathrm{CO}_{2}$ washout by expanding collapsed alveoli and recruiting lung volume; (2) reducing the work of breathing; and (3) preventing apnea by stabilizing the upper airways and chest wall, particularly in ex pre-term babies. NPPV is extensively delivered by piston-driven or turbine ventilators. During NPPV, patients can be completely controlled by the ventilator (total controlled ventilatory support) or the patient's spontaneous inspiratory effort triggers (assisted ventilatory support) the ventilator to provide a variable volume (volume-targeted ventilation) or pressure (pressure-targeted ventilation).

During pressure-targeted ventilation the patient receives a pressure-supported flowcycled breath (pressure support ventilation) or a time-cycled breath (assisted pressure-controlled ventilation). Unlike NCPAP, NPPV theoretically allows improved respiratory muscle unloading, alveolar recruitment, oxygenation, and $\mathrm{CO}_{2}$ washout improvement, but patient-ventilator asynchrony may become a major issue, leading to NPPV treatment failure. Two recent physiological papers [11-12] demonstrated the effectiveness of NPPV in reducing inspiratory effort as evaluated by esophageal and transdiaphragmatic pressure-time product and esophageal tidal swings in children with ARF. In addition, the application of NPPV via nasal and/or facial mask was associated with significant improvement in breathing pattern and gas exchange.

\subsubsection{Hypoxemic Acute Respiratory Failure}

Hypoxemic respiratory failure is characterized by hypoxemia associated with low or normal levels of partial pressure of carbon dioxide $\left(\mathrm{PaCO}_{2}\right)$ in the blood. The underlying predominant mechanism is uneven or mismatched ventilation-perfusion in regional lung units. Hypoxemic respiratory failure mainly occurs in disorders characterized by parenchymal pathologies, such as bacterial and viral pneumonia, as well as in lower airway obstruction, such as bronchiolitis and status asthmaticus. 
Streptococcus pneumoniae is the most common agent responsible for pneumonia, although other microorganisms can play an important role. Pneumonia produces a reduction in lung volume, due to consolidation and/or atelectasis, leading to reduced lung compliance. Bronchiolitis occurs mainly in children of less than 2 years of age and respiratory syncytial virus is estimated to be the most frequent etiological cause. Bronchiolitis causes an increase in airway resistance with dynamic lung hyperinflation, but this pathology often also involves the lung interstitium, with reduced lung volume and atelectasis. Both pneumonia and bronchiolitis can lead to acute respiratory distress syndrome (ARDS).

\subsubsection{Hypercapnic Acute Respiratory Failure}

The ability to breathe spontaneously is the result of a balance between neurological mechanisms controlling ventilation (central respiratory drive), together with ventilatory muscle pump power, on the one hand, and the respiratory load (resistive and elastic load), determined by the airway, lung, and thoracic elastance, on the other hand. In healthy children, the central respiratory drive and the ventilatory muscle pump exceed the respiratory load thus maintaining adequate spontaneous ventilation. However, if the force generated by the respiratory muscles pump (fatigue or weakness), or central respiratory drive is too low and/or the respiratory load is too high, the resulting alveolar ventilation may be inadequate, thus leading to hypercapnia [13]. This phenomenon can be "acute", when the imbalance is caused by an acute condition (e.g., acute exacerbation of an asthmatic patient), or "chronic", when the surge is slow during the course of a disease (e.g., a neuromuscular disease) $[14,15]$.

\subsubsection{When Should Noninvasive Respiratory Support be Used?}

In adult patients, as mentioned previously, NRS has been proposed in two different contexts: (1) as a preventive or "prophylactic" application in postoperative patients to prevent ARF and extubation failure in patients at risk; and (2) as a "curative" application, once ARF occurs, to improve respiratory function and avoid endotracheal intubation. Unlike adults, to our knowledge no papers have been published to date in the pediatric literature on the use of NRS in the postoperative period. As a curative application, NRS should be initiated according to: (1) clinical signs: moderateto-severe dyspnea and/or tachypnea (defined as a respiratory rate $>$ the 75 th percentile depending on the age of the patient); and (2) gas exchange derangement: hypoxemia [defined as a fraction of inspired oxygen $\left(\mathrm{FiO}_{2}\right)>0.5$ to obtain a saturation of peripheral oxygen $\left(\mathrm{SpO}_{2}\right)>94 \%$ ] and/or respiratory acidosis (defined as $\mathrm{pH}<$ 7.35). Possible contraindications for NRS are: life-threatening hypoxemia; upper airway obstruction; vomiting; cough or impaired gag reflex; facial surgery, facial trauma, or facial deformity; Glasgow Coma Scale $<10$; hemodynamic instability requiring inotropes or vasopressors, or cardiac arrhythmia; and cyanotic congenital heart disease. NRS should not be started in more severe ARF in the presence of: (1) clinical 
signs of exhaustion (active contraction of the accessory muscles of respiration with paradoxical abdominal and thoracic motion); and (2) a $\mathrm{PaO}_{2}: \mathrm{FiO}_{2}$ ratio $<150 \mathrm{mmHg}$ and/or $\mathrm{PaCO}_{2}>55 \mathrm{mmHg}$; and (3) $\mathrm{pH}<7.30$.

\subsubsection{Noninvasive Respiratory Support in Clinical Settings}

There are no data describing how to initiate NRS in children. The current knowledge is mainly based on the direct experience of clinicians working in the field, and a variety of routines are applied. Pressure target mode is by far the most common ventilatory modality in pediatric intensive care units (PICUs) [16].

However, when applying NRS via a nasal route (i.e., nasal prongs), the high nasal resistance must be taken into account. In NCPAP, PEEP pressures between 4 and $8 \mathrm{~cm} \mathrm{H}_{2} \mathrm{O}$ are safe and not associated with adverse hemodynamic effects. Of note, when NCPAP is delivered by helmet, a high flow system should be used to prevent $\mathrm{CO}_{2}$ rebreathing (minimum flow rate: $30 \mathrm{~L} / \mathrm{min}$ ) [17]. A ventilator should never be connected to a helmet in CPAP mode.

\subsubsection{Ventilators}

Administering noninvasive therapeutic positive pressure ventilation is achievable through high-pressure gas flow and piston-driven or turbine ventilators. When the patient spontaneously initiates ventilation in pressure target modes, the machine is triggered and the inspiratory effort is immediately followed by the administration of a support pressure by the ventilator to reach a preset inspiratory pressure. This assumes perfect patient-machine interaction and minimal air leakage between the patient's airway and the interfaces to minimize the delay between the patient's efforts and the activation of the trigger to avoid asynchronies. Ineffective triggering and auto-triggering has been shown to be the leading cause of NRS failure due to discomfort, hyperventilation, and dynamic hyperinflation in adults [18].

The ventilator then cycles to the expiratory phase when the inspiratory flow decreases to a preset value (usually $25 \%$ of the peak inspiratory flow), as observed in pressure support ventilation, or when the patient reaches a preset inspiratory time, as observed in assisted pressure-controlled ventilation. However, during pressure support ventilation, the presence of any leak may cause the ventilator to fail to cycle to expiration causing a prolonged inspiratory time ("inspiratory hung-up"). This may cause expiratory effort, hyperinflation, discomfort, and fatigue, leading to adverse respiratory or hemodynamic consequences and NRS failure [19]. The importance of safe, comfortable, and well-fitting interfaces is mandatory to achieve success in NRS not only in pediatric patients.

In adults, ineffective inspiratory effort and double-triggering are the most common types of asynchrony leading to patient discomfort [18], whereas in children auto-triggering has been recently shown to be the primary cause of difficult patient-ventilator interaction [20]. 
Ueno and colleagues [21] investigated how different ventilators cope with different interface leaks. They tested three "home use" ventilators (Respironics BiPAP Vision, Respironics Trilogy 100, Murrysville, PA, USA, and Carina, Draeger, Lubeck, Germany), and two intensive care unit (ICU) ventilators (Puritan Bennett 840 Ventilator System, Covidien, Mansfield, MA, USA, and Evita XL, Draeger, Lubeck, Germany) at various positive pressure settings and leak sizes, finding that home ventilators performed better at compensating for small and medium-sized leaks. In a single-center observational prospective study, Muñoz-Bonet and colleagues [22] investigated how an ICU ventilator (Evita 2 Dura, Draeger, Lubeck, Germany) equipped with leak compensation software coped with air leaks in different modes of ventilation of pediatric patients with ARF. The study showed the effectiveness of the software in different subsets of infants and children in reducing patient-machine asynchrony. In several studies, the augmentation of trigger sensitivity, clinical observation, and the presetting of limited inspiratory time are efficient measures addressed at preventing asynchrony [8].

\subsection{Interfaces}

In the clinical setting, several different interfaces can be used to deliver NRS: nasal prongs, nasal masks, oronasal masks, and full-face masks. Recently, the use of a helmet has emerged and has reached immediate popularity, proving to be an effective and comfortable means of delivering positive airway pressure noninvasively [8,23], avoiding skin breakdown and other mask adverse effects [24]. Nasal prongs are typically used for the youngest patients: when directly inserted in the patient's nostrils, they are more effective for obligate nose breathers, such as newborns and young infants up to 1 year of life, in delivering continuous positive airway pressure. Normally this interface is easily kept in place without any other device, but nasal prongs are poorly tolerated for longer periods, are highly flow resistive (due to nasal anatomical resistance, to small airway resistance, and the high propensity of these patients to have hypertrophic adenoids and tonsils), and are easily obstructed by an excess of nasal secretions. Quite common side effects are bleeding, skin lesions, and nasal dryness due to airflow and nostril obstruction $[3,16]$.

A good alternative for patients of the same age is a nasal mask. It is a small, soft and transparent mask which completely covers the nasal surface, and through which it is possible to achieve proper fitting, minimal dead space, and minimal leaks; it is therefore useful for delivering both NCPAP and NPPV. Skin lesions and mucosal breakdown are the more frequent side effects. Limitations in the use of a nasal mask include larger pressure drops due to the mouth opening [3] and the impracticability of nasogastric tube positioning [25]. In older patients (the so-called nonobligate nose breathers), oronasal masks covering both nose and mouth are more effective in minimizing air leaks and thus preserving the necessary pressurization of the respiratory system. The need for tightened straps to keep the mask in place is the main cause for patient discomfort and skin irritation. The recent introduction in the market of a 
full-face mask covering the entire facial surface is very promising, although no studies have been published in the pediatric population. Nasal masks, oronasal, and fullface masks need to be well-fitted to deal with leaks and discomfort, important problems that are not overcome by the use of hydrocolloid protection and that are exacerbated by facial deformities, facial trauma, and pre-existing facial lesions [8]. The recently introduced helmet is also promising in pediatric clinical practice. While its use is well established in the adult population [26,27] a few studies assessed its feasibility and effectiveness in pediatrics, both in PICUs and clinical wards, especially in acute bronchiolitis $[23,28,29]$. The helmet is a soft, transparent, pressurized plastic chamber that encloses the head and neck. It is easily applied in both nose and mouth breathers and in a wide range of ages and anatomical variations. Moreover, it warrants good patient-environment interaction, comfort, and reduced need for sedation in the treatment of ARF. Furthermore, the helmet allows good clearance of secretions, allows the patient to speak and swallow, and it is well tolerated for prolonged periods of application.

\subsection{Predictive Factors of Noninvasive Respiratory Support Failure}

NRS constitutes an alternative treatment for early pediatric ARF provided that tracheal intubation is not delayed when considered necessary. One of the major challenges during NRS is to identify the early prognostic signs of treatment failure. In a 4-year study, Muñoz-Bonet and colleagues [5] investigated several predictive factors of NRS failure in children from 1 month to 16 years of age with moderate-tosevere ARF. NRS failure was defined as the need for tracheal intubation. NRS was applied in 47 consecutive patients and failed in $9(19.1 \%)$ due to the progression of ARF. Younger age, diagnosis of ARDS, and chest X-ray worsening at $24 \mathrm{~h}$ from the beginning of NRS correlated with treatment failure. The authors could also show that the association between mean airway pressure $>11.5 \mathrm{~cm} \mathrm{H}_{2} \mathrm{O}$ and $\mathrm{FiO}_{2}>0.6$ was predictive of NRS failure in nearly $80 \%$ of children.

In a prospective observational study, Lum and colleagues [30] investigated the factors that predict outcome of NRS in critically ill children admitted to a multidisciplinary PICU of a university hospital in Kuala Lumpur, Malaysia. Out of 278 children (average age: 8.7 months) with ARF and treated with NRS, 129 received NRS as the sole ventilatory support, 98 were treated with NRS to prevent extubation failure and 48 because of postextubation ARF. Interestingly, $71.2 \%$ of children had an underlying chronic disease, probably reflecting the typical PICU population of a developing country. Overall, NRS avoided intubation in more than $75 \%$ of children. During this study, a high pediatric risk of mortality (PRISM II) score, the presence of sepsis, an abnormal respiratory rate, and a high requirement of $\mathrm{FiO}_{2}$ at $\mathrm{NRS}$ initiation were found to be independent predictive factors of NRS failure. Worsening respiratory failure and septic shock were the two leading causes of failure of NRS. The authors concluded that NRS represents an effective strategy to prevent 
tracheal intubation and for rapid discharge to the ward where respiratory treatment can be continued.

A high PRISM II score, a high respiratory rate, the need for oxygen, and the presence of sepsis at initiation of NRS should suggest closer monitoring to prevent NRS failure. At the Great Ormond Street Hospital in London, 163 patients aged between 1 month and 18 years who received NRS during the 7-year study period, were evaluated to determine whether physiological parameters and an underlying condition predict NRS success [31]. Eighty-three children received NRS as firstline intervention to avoid intubation and $64 \%$ of them succeeded. Those who failed showed higher $\mathrm{FiO}_{2}(0.56$ vs. $0.47, p=0.038)$, higher respiratory rate (53.3 vs. 43.3 breaths/min, $p=0.012)$, and lower $\mathrm{pH}(7.26$ vs. $7.34, p=0.032)$ before NRS was started and higher $\mathrm{FiO} 2$ requirements once NRS was applied. Eighty patients were started on NRS to prevent postextubation failure and $60 \%$ were treated successfully. Those individuals who failed showed significantly higher systolic and diastolic blood pressure $2 \mathrm{~h}$ after NRS start (104 vs. $77.9 \mathrm{mmHg}, p=0.001$ and 64.5 vs. $54.1 \mathrm{mmHg}, p=0.037$ ), probably representing a stress response in the worsening child.

Interestingly, patients on CPAP were more likely to avoid intubation when compared with those on bilevel positive airway pressure in both groups (first-line elective and postextubation NRS). Looking at the underlying conditions, the authors demonstrated that children with a primary respiratory disease who were treated with NRS as the first-line treatment avoided intubation in 30/36 cases (83\%), while those with an underlying oncological disease showed a much lower success rate $(8 / 23$ cases, $35 \%)$. The presence of sepsis further decreased the rate of success in the oncological group (3/15 cases, $20 \%)$. Patients with a primary respiratory illness were also more likely to avoid reintubation after extubation (27/33 cases, $82 \%$ ). The authors concluded that tachypnea and acidosis prior to establishing NRS treatment and oxygen requirement pre- and post-NRS are the strongest predictive factors for treatment failure when NRS is used as the first-line treatment to prevent intubation. In contrast, when NRS is used to avoid reintubation, the most important predictive factor for reintubation is persistent hypercapnia after NRS initiation.

\subsection{Conclusions}

The use of NRS in the pediatric population has become an option in the last few years, and has been increasingly applied. In general, the evidence supporting its use in infants and children with ARF is still limited and the identification of the right patient, the right time of application, and the appropriate setting is still lacking, as well as universally accepted guidelines. However, the most recent physiological and randomized studies indicate that the early application of NRS can ameliorate the breathing pattern and gas exchange, and reduces respiratory muscle loading. The effects of NRS on more complex outcomes require further investigation. 
To date, CPAP delivered noninvasively via a nasal mask or helmet could be considered as a first-line respiratory treatment in infants and children with mildto-moderate ARF. NPPV applied via a facial mask probably represents the technique of choice in moderate-to-severe acute respiratory disorders, but the patientmachine interaction may become a relevant problem, particularly in younger infants. Furthermore, it is important to rely on well-trained medical and nursing staff at all times, and to apply NRS only in an ICU setting which also has appropriate cardiorespiratory monitoring.

The type of equipment and the specific ventilator settings that should be chosen remain a matter of debate. The specific equipment available for therapy evolves more rapidly with industry capability rather than with clear indications available from scientific trials.

Further studies are urgently needed to determine the criteria to initiate NPPV according to the disease profile and the age of the patient.

\section{References}

1. Orlowski JP, Ellis NG, Amin NP et al (1980) "Complications of airway intrusion in 100 consecutive cases in a pediatric ICU". Critical Care Medicine 8:324-331

2. Craven DE, Kunches LM Kilinsky V (1986) "Risk factors for pneumonia and fatality in patients receiving continuous mechanical ventilation". American Journal of Review of Respiratory Disease 133:792-796

3. Nava S, Navalesi P, Carlucci A (2009) Non-invasive ventilation. Minerva Anestesiologica $75: 31-36$

4. Chidini G, Gregoretti C, Pelosi P, Calderini E (2010) "Non-invasive respiratory support in children". eBook edition of Treatment Strategies-Paediatrics Cambridge Research Centre. Available online at: http://viewer.zmags.com/publication/18ab08e6"http://viewer.zmags.com/ publication/18ab08e6"http://viewer.zmags.com/publication/18ab08e6

5. Muñoz-Bonet JI, Flor-Macián EM, Roselló PM et al (2010) "Predictive factors for the outcome of noninvasive ventilation in pediatric acute respiratory failure". Pediatric Critical Care Medicine 11:675-680

6. Lum LC, Abdel-Latif ME, De Bruyne JA et al (2011) "Noninvasive ventilation in a tertiary pediatric intensive care unit in a middle-income country". Pediatric Critical Care Medicine 12:e7-e13

7. Lazner MR, Basu AP, Klonin H (2012) "Non-invasive ventilation for severe bronchiolitis: analysis and evidence". Pediatric Pulmonology. Published online in Wiley Online Library (wileyonlinelibrary.com) DOI 10.1002/ppul.22513

8. Chidini G, Calderini E, Pelosi P (2010) "Treatment of acute hypoxemic respiratory failure with continuous positive airway pressure delivered by a new pediatric helmet in comparison with a standard full face mask: a prospective pilot study". Pediatric Critical Care Medicine 11:502-508

9. Essouri S, Durand P, Chevret L et al (2008) "Physiological effects of noninvasive positive ventilation during acute moderate hypercapnic respiratory insufficiency in children". Intensive Care Medicine 34:2248-2255

10. Dohna-Schwake C, Stehling F et al (2011) "Non-invasive ventilation on a pediatric intensive care unit: Feasibility, efficacy, and predictors of success." Pediatric Pulmonology 46(11):1114-1120 
11. Essouri S, Durand P, Chevret L et al (2011) "Optimal level of nasal continuous positive airway pressure in severe viral bronchiolitis". Intensive Care Medicine 37:2002-2007

12. Stucki P, Perez MH, Scalfaro P et al (2009) "Feasibility of non-invasive pressure support ventilation in infants with respiratory failure after extubation: a pilot study". Intensive Care Medicine 35:1623-1627

13. Hertzog JH, Costarino AT Jr (1996) "Nasal mask positive pressure ventilation in paediatric patients with type II respiratory failure". Paediatric Anaesthesiology 6:219-224

14. Piastra M, Antonelli M, Caresta E et al (2006) "Noninvasive ventilation in childhood acute neuromuscular respiratory failure: a pilot study". Respiration 496:791-798

15. Niranjan V, Bach JR (1998) "Noninvasive management of pediatric neuromuscular ventilatory failure". Critical Care Medicine 26:2061-2065

16. Calderini E, Chidini G, Pelosi P et al (2010) "What are current indications for non invasive ventilation in children?". Current Opinion in Anaesthesiology 23(3):368-374

17. Patroniti N, Foti G, Manfio A et al (2003) "Head Helmet versus Face Mask for Non Invasive CPAP: a physiological study". Intensive Care Medicine 29:1680-1687

18. Thille AW, Rodriguez P, Cabello B et al (2006) "Patient-ventilator asynchrony during assisted mechanical ventilation". Intensive Care Medicine 32(10):1515-1522

19. Calderini E (1999) "New insights in mechanical ventilation for pediatric patients". Intensive Care Medicine 25(10):1194-1196

20. Fauroux B, Leroux K, Desmarais G et al (2008) "Performance of ventilators for noninvasive positive-pressure ventilation in children”. European Respiratory Journal 31(6):1300-1307

21. Ueno Y, Nakanishi N, Oto J et al (2011) "Effects of leakage on ventilator performance during noninvasive positive pressure ventilation: a bench study". Respiratory Care 56:1758-1764

22. Muñoz-Bonet JI, Flor-Macián EM, Roselló PM et al (2010) "Noninvasive ventilation in pediatric acute respiratory failure by means of a conventional volumetric ventilator". World Journal of Pediatrics 6:323-330

23. Chidini G, Calderini E, Cesana BM et al (2010) Noninvasive Continuous Positive Airway Pressure in Acute Respiratory Failure. Helmet versus Facial Mask. Pediatrics 128:e330-e338

24. Fauroux B, Lavis JF et al (2005) "Facial side effects during noninvasive positive pressure ventilation in children." Intensive Care Medicine 31(7):965-969

25. Nørregaard O (2002) "Non invasive ventilation in children". European Respiratory Journal 20:1332-1342

26. Isgrò $\mathrm{S}$, Zanella $\mathrm{A}$, Sala $\mathrm{C}$ et al (2005) "Continuous flow biphasic positive airway pressure by helmet in patients with acute hypoxic respiratory failure: effect on oxygenation". Intensive Care Medicine 36(10):1688-1694

27. Milan M, Zanella A, Isgrò S et al (2011) "Performance of different continuous positive airway pressure helmets equipped with safety valves during failure of fresh gas supply". Intensive Care Medicine 37(6):1031-1035

28. Javouhey E, Barats A, Richard N et al (2008) "Non-invasive ventilation as primary ventilatory support for infants with severe bronchiolitis". Intensive Care Medicine 34:1608-1614

29. Yanez LJ, Yunge M, Emilfork M et al (2008) "A prospective, randomized, controlled trial of non-invasive ventilation in pediatric acute respiratory failure". Pediatric Critical Care Medicine 9:484-489

30. Lum LC, Abdel-Latif ME, De Bruyne JA et al (2011) "Noninvasive ventilation in a tertiary pediatric intensive care unit in a middle-income country". Pediatric Critical Care Medicine 12:e7-e13

31. James CS, Hallewell CP, James DP et al (2011) "Predicting the success of non- invasive ventilation in preventing intubation and re-intubation in the paediatric intensive care unit". Intensive Care Medicine 37:1994-2001 